\title{
COMPORTAMENTO PRÓ-AMBIENTAL NA AGRICULTURA E IMPLICAÇÕES À EDUCAÇÃO AMBIENTAL: REVISÃO DE LITERATURA
}

\author{
Ana Paula da Silva Siqueira ${ }^{1}$ \\ Bianca Ramos Meira ${ }^{2}$ \\ Tiago Teixeira da Silva Siqueira ${ }^{3}$ \\ Ednéia Aparecida de Souza Paccola ${ }^{4}$ \\ Rute Grossi-Milani ${ }^{5}$
}

Resumo: Esse estudo visou analisar as produções científicas sobre o comportamento pró-ambiental na agricultura e descrever os principais fatores pessoais e ambientais associados. Foram utilizadas as palavras-chave: comportamento pró-ambiental e proenvironmental behavior nas principais bases de dados disponíveis. As principais condições associadas ao comportamento pró-ambiental foram de natureza intrínsecas, como os valores biosféricos, consciência ambiental, orientação para o futuro, autoeficácia e conhecimento. A promoção de políticas públicas de incentivo ao comportamento pró-ambiental, aliadas à Educação Ambiental propulsora de valores ambientais, estimulam às práticas agrícolas sustentáveis.

Palavras-chave: Agricultura Sustentável; Psicologia Ambiental; Comportamento Ecológico; Educação Ambiental; Saúde Ambiental.

Abstract: This study aimed to analyze scientific productions on pro-environmental behavior in agriculture and to describe the main associated personal and environmental factors. The keywords were used: pro-environmental behavior and proenvironmental behavior in the main available databases. The main conditions associated with pro-environmental behavior were of an intrinsic nature, such as biospheric values, environmental awareness, orientation for the future, self-efficacy and knowledge. The promotion of public policies to encourage pro-environmental behavior, combined with environmental education that generates knowledge and promotes environmental values, encourages sustainable agricultural practices.

Keywords: Sustainable Agriculture; Environmental Psychology; Ecological Behavior; Environmental Education; Environmental Health.

\footnotetext{
1 Universidade Cesumar; Faculdade Unyleya. E-mail: contatopsianasiqueira@gmail.com, Link para o Lattes: http://lattes.cnpq.br/3073686364870809

2 Universidade Estadual de Maringá. Email: bianca.rmeira@hotmail.com. Link para o Lattes: http://lattes.cnpq.br/8527226743705523

${ }^{3}$ National Research Institute for Agriculture, Food and Environment. E-mail: tiago.teixeira.dasilva.siqueira@gmail.com. Link para o Lattes: http://lattes.cnpq.br/2102738882998313

${ }^{4}$ Universidade Cesumar. E-mail: edneia.paccola@unicesumar.edu.br. Link para o Lattes: http://lattes.cnpq.br/5090759600495959

${ }_{5}^{5}$ Universidade Cesumar. E-mail: rutegrossimilani@gmail.com.

Link para o Lattes: http://lattes.cnpq.br/8844448878404124
} 


\section{Introdução}

A agricultura é essencial para a existência humana e representa uma das principais formas de interação pessoa-ambiente (LIMA, 2017). Historicamente, ela desempenhou influência fundamental sobre a quantidade de alimentos disponíveis e foi imprescindível para aumentar a densidade populacional do planeta (LIMA, 2017).

As práticas agrícolas começaram há cerca de dez mil anos atrás pelos homens pré-históricos, o que tornou a oferta de alimento mais acessível e previsível. Essas práticas vêm sendo modificadas pela apropriação de novas tecnologias no decorrer do processo evolutivo (RAMANKUTTY et al. 2018). No entanto, o aumento da qualidade de vida em detrimento do consumo exorbitante de recursos ambientais tem impactado negativamente o meio ambiente, ocasionando inúmeros problemas ambientais, como as alterações climáticas e a deterioração dos recursos naturais (KLÖCKNER, 2013). A forma pautada no agronegócio tem gerado inúmeros problemas ambientas e sociais, colocando-o entre os maiores geradores de gases de efeito estufa, segundo o relatório da Organização e Cooperação para o Desenvolvimento Econômico (ORGANISATION FOR ECONOMIC CO-OPERATION AND DEVELOPMENT OCDE, 2017).

Diante dessa problemática, o Programa das Nações Unidas para o Desenvolvimento (PNUD), em 2015, propôs os Objetivos de Desenvolvimento Sustentável (ODS). Um desses objetivos se refere à erradicação da fome, alcance da segurança alimentar e melhoria nutricional. Assim como, promover a adoção de sistemas sustentáveis de produção agrícola. Dessa maneira, as práticas sustentáveis na agricultura se propõem a melhorar a produção e a segurança alimentar, além de abrangerem a dimensão socioambiental (ZEWELD et al., 2017; SZUMELDA, 2019).

A proposta de desenvolvimento sustentável constitui grande desafio mundial, envolve mudanças desde a adoção de estilo de vida sustentável, bem como, a criação e efetivação de políticas públicas pelos governos, em conjunto com a sociedade, e que garantam a sustentabilidade ambiental, a viabilidade econômica e a qualidade de vida de forma integrada (LIMA, 2003; ZACARIAS; HIGUCHI, 2017). Destarte, defronte as necessidades atuais de preservação ambiental criaram-se novas perspectivas para a agricultura, exigindo transformações na relação pessoa-ambiente (GUEVARA-HERNANDEZ, 2017). Portanto, para se atingir o desenvolvimento sustentável, faz-se necessário o envolvimento dos indivíduos em comportamentos pró-ambientais, principalmente no setor agrícola (CONWAY; BARBIER, 2013; WU et al., 2013).

Diante desse cenário, a Psicologia Ambiental vem contribuir para as discussões a respeito da inter-relação pessoa-ambiente, considerando os vínculos cognitivos e afetivos que emergem dessa interação, no processo de transformar e ser transformado pelo ambiente e como esses fatores influenciam no comportamento humano (BEZERRA; FEITOSA, 2018). O termo comportamento, na Psicologia Ambiental, possui diferentes qualificadores de 
acordo com a literatura sobre a inter-relação entre indivíduos e meio ambiente tais como: "comportamento ambientalmente responsável", "comportamento ecológico", "comportamento ambientalmente amigo", "comportamento próambiental". No entanto, Martínez-Soto (2004) ressalta que esses termos não são definidos satisfatoriamente, visto que existem várias formas de abordagem. Corral-Verdugo (2000) conduziu uma revisão de literatura com o intuito de comparar as investigações, a fim de evitar conclusões errôneas sobre estes tipos de comportamento, e ter uma definição mais precisa para 0 desenvolvimento de objetivos em projetos na área da Educação Ambiental. O autor, então, adotou a terminologia comportamento pró-ambiental definindo-o como: "O conjunto de ações dirigidas, deliberadas e efetivas que respondem a requerimentos sociais e individuais e que resultam na proteção do meio ambiente" (CORRAL-VERDUGO, 2000, p. 471). Portanto, o comportamento pró-ambiental tem sido um dos temas de maior interesse em Psicologia Ambiental.

De acordo com Hackett (1993), o comportamento pró-ambiental é em grande parte função das preocupações ambientais, que por sua vez são baseadas na consciência pessoal e social. Nos últimos anos, o estudo psicológico do ambientalismo, isto é, a preocupação de um indivíduo pela preservação, restauração ou melhoria do ambiente natural, tornou-se cada vez mais presente (SWAMI et al., 2010; CLAYTON et al., 2016; BERNSTEIN; SZUSTER, 2019; ALCOCK et al., 2020). Diversas variáveis são utilizadas para entender o comportamento pró-ambiental, entre elas: a motivação, as crenças ambientais, a consciência ambiental, o altruísmo, o nível informacional e a percepção ambiental. E a Educação Ambiental pode desenvolver intervenções que potencializam o comportamento pró-ambiental (CHIERRITO-ARRUDA et al., 2018), inclusive na agricultura por meio de práticas agrícolas sustentáveis.

À face do exposto, o presente estudo visa analisar as produções científicas sobre o comportamento pró-ambiental na agricultura, descrever os principais fatores pessoais e ambientais associados, que podem servir como subsídio ao planejamento de intervenções em Educação Ambiental e ao planejamento de políticas públicas.

\section{Materiais e métodos}

A presente revisão de literatura utilizou três indexadores: American Psychological Association (PsycINFO), Scientific Eletronic Library Online (SciELO) e Web of Science (WoS). Foram usadas as terminologias de busca: comportamento pró-ambiental e pro-environmental behavior. Optou-se por empregar os termos abrangentes, visto que, ao inserir a palavra agricultura na busca eletrônica não foram identificados artigos na maioria das bases pesquisadas. Em seguida, selecionou-se manualmente os estudos que enfocaram o comportamento pró-ambiental na agricultura. Os procedimentos aplicados nesta revisão foram inspirados na metodologia PRISMA - Preferred 
Reporting Items for Systematic Reviews and Meta-Analyses (MOHER et al. 2015).

Os critérios de elegibilidade consistiram em todos os artigos já publicados e disponíveis na íntegra, de 1999 até o ano de 2019, sobre comportamento pró-ambiental. Foram excluídos artigos de revisão de literatura, pesquisas documentais, anais de congressos e textos não publicados em formato de artigo. A busca inicial com o emprego das terminologias comportamento pró-ambiental e pro-environmental behavior mostrou 1638 resultados, destes 1.176 eram artigos científicos. Após a leitura dos títulos e resumos do material coletado, aplicando-se os critérios de elegibilidade e de $100 \%$ de concordância entre os autores, selecionaram-se 26 artigos.

\section{Resultados e discussão}

À vista da problemática ambiental, a busca pelo desenvolvimento sustentável faz-se primordial, que por sua vez, perpassa pelo comportamento pró-ambiental. Os dados obtidos a partir dos artigos selecionados demonstram que há preocupação da comunidade científica com a temática (Tabela 1).

Tabela 1: Número de artigos publicados nas bases de dados PsycINFO, SciELO e WoS sobre o comportamento pró-ambiental na agricultura.

\begin{tabular}{|c|c|c|c|c|}
\hline Ano & Autor & Periódico & Participantes (n) & País \\
\hline 1999 & $\begin{array}{c}\text { Pyrovetsi e } \\
\text { Daoutopoulos }\end{array}$ & Journal of Environmental Management & 337 & Grécia \\
\hline 2010 & Swami et al. & Personality and Social Sciences & 203 & Inglaterra \\
\hline 2012 & Schupp e Sharp & Agriculture and Human Values & 1750 & Estados Unidos \\
\hline 2013 & Wu et al. & Plos One & 229 & Canadá \\
\hline 2013 & Klöckner & Global Environmental Change & 56 & Noruega \\
\hline 2013 & Bjornlund et al. & Society \& Natural Resources & 1170 & Canadá \\
\hline 2014 & Price e Leviston & Journal of Rural Studies & 422 & Austrália \\
\hline 2015 & Morgan et al. & Journal of Environmental Psychology & 551 & Austrália \\
\hline $\begin{array}{l}2015 \\
2015\end{array}$ & $\begin{array}{l}\text { Honig et al. } \\
\text { Arora et al. }\end{array}$ & $\begin{array}{c}\text { Land Use Policy } \\
\text { Journal of Behavioral and Experimental } \\
\text { Economics }\end{array}$ & $\begin{array}{l}38 \\
46\end{array}$ & $\begin{array}{l}\text { África do sul } \\
\text { Argentina }\end{array}$ \\
\hline $\begin{array}{l}2016 \\
2016\end{array}$ & $\begin{array}{l}\text { Soncini et al. } \\
\text { Nguyen et al. }\end{array}$ & $\begin{array}{c}\text { Retratos de Assentamentos } \\
\text { Journal of Retailing and Consumer } \\
\text { Services }\end{array}$ & $\begin{array}{c}64 \\
703\end{array}$ & $\begin{array}{l}\text { Brasil } \\
\text { Vietnã }\end{array}$ \\
\hline 2016 & Hyland et al. & Agriculture and Human values & 286 & Inglaterra \\
\hline 2016 & Gould et al. & Environmental Management & 1201 & Estados Unidos \\
\hline 2016 & García-Lorente et al. & Sustainability & revisão & Itália \\
\hline $\begin{array}{l}2017 \\
2017\end{array}$ & $\begin{array}{l}\text { Adnan et al. } \\
\text { Bijani et al. }\end{array}$ & $\begin{array}{l}\text { Land Use Policy } \\
\text { International Soil and Water } \\
\text { Conservation Research }\end{array}$ & $\begin{array}{l}\text { revisão } \\
9621\end{array}$ & $\begin{array}{l}\text { Malásia } \\
\text { Irã }\end{array}$ \\
\hline
\end{tabular}

Continua... 
,...continuação.

\begin{tabular}{|c|c|c|c|c|}
\hline Ano & Autor & Periódico & Participantes (n) & País \\
\hline 2017 & Brown e Roper & $\begin{array}{l}\text { Australian journal of agricultural and } \\
\text { resource economics }\end{array}$ & 2839 & Nova Zelândia \\
\hline 2017 & Zeweld et al. & Journal of Environmental Management & revisão & Etiopia \\
\hline 2018 & Benedetti e Laureti & Journal of Cleaner Production & 50.000 & Itália \\
\hline 2018 & $\begin{array}{l}\text { Chierrito-Arruda et } \\
\text { al. }\end{array}$ & Ambiente \& Sociedade & 14 & Brasil \\
\hline 2018 & Fang et al. & Sustainability & 384 & Taiwan \\
\hline 2019 & Foguesatto et al. & Journal of Cleaner Production & 172 & Brasil \\
\hline 2019 & Lazaroiu et al. & Frontiers in Public Health & revisão & Vários \\
\hline 2019 & Pungas & Journal of Rural Studies & 10 & Estônia \\
\hline 2019 & $\begin{array}{l}\text { Schinaider e } \\
\text { Talamini }\end{array}$ & $\begin{array}{l}\text { Revista Brasileira de Gestão e } \\
\text { Desenvolvimento Regional }\end{array}$ & 105 & Brasil \\
\hline
\end{tabular}

Fonte: Autores (2020)

Os comportamentos pró-ambientais mais frequentes encontrados estavam relacionados a aspectos simples do gerenciamento de resíduos, já comportamentos mais complexos que envolvessem mudanças no estilo de vida, como o consumo consciente, ocorreram com menor frequência. Esse fato é explicitado por Gould et al. (2016), ao discutirem que a natureza dos comportamentos relacionados à gestão de resíduos é concreta, ou seja, são atos visíveis, e apoiados por infraestrutura, ações simples de serem efetuadas e não exigem mudanças substanciais no estilo de vida.

Apesar de considerável número de pesquisas investigarem as relações entre construtos psicológicos e comportamentos pró-ambientais urbanos, estudos com foco em comportamentos pró-ambientais na agricultura são menos comuns (MORGAN et al. 2015). Esse fenômeno foi observado nesta pesquisa em que foram encontrados apenas 26 artigos empíricos em relação ao comportamento pró-ambiental na agricultura, o que corresponde a apenas $2,21 \%$ das publicações sobre o comportamento pró-ambiental encontradas nesta pesquisa. Para Price e Leviston (2014), a falta de quadros teóricos adequados limita as pesquisas sobre os comportamentos pró-ambientais dos agricultores, visto que há pouca integração entre as ciências na literatura científica a respeito da adoção de práticas agrícolas.

Conforme demonstrado na Tabela 1, o ano com o maior número de publicações foi 2016, o que é bastante positivo, pois demonstra o relativo aumento da preocupação em relação às práticas sustentáveis na agricultura. Esse fenômeno é descrito Morgan et al. (2015) que apontam a crescente conscientização e preocupação com a degradação ambiental, que tem desencadeado o aumento do interesse pelos fatores psicológicos e barreiras subjacentes ao comportamento pró-ambiental. 
As principais temáticas referentes ao comportamento pró-ambiental na agricultura encontradas nos artigos foram: a forma de produção, água, inovação e tipo de propriedade (Tabela 2). A maior parte dos artigos teve como objetivo a análise de quais fatores influenciavam a escolha da forma de produção ser sustentável ou não. De acordo com Guevara-Hernandez (2017), o aumento da preocupação em relação à forma de produção sustentável se deve à crescente conscientização com a segurança alimentar, a degradação ambiental e a mudança climática, o que deu origem a novas ideias e abordagens para o desenvolvimento agrícola.

Tabela 2: Porcentagem das temáticas relacionadas ao comportamento pró-ambiental na agricultura.

\begin{tabular}{cc}
\hline Temáticas & Porcentagem \\
\hline Formas de produção & $64,70 \%$ \\
Água & $17,60 \%$ \\
Inovação & $11,70 \%$ \\
Tipo de propriedade & $5,80 \%$ \\
\hline
\end{tabular}

Fonte: Autores (2020)

As práticas agrícolas sustentáveis caracterizam-se como ambientalmente não-degradantes, tecnicamente apropriadas e economicamente viáveis (FAO, 1995). Essas práticas são direcionadas para o uso eficiente dos recursos naturais e envolvem diversas práticas, tais como: uso de consorciação, rotação de culturas e manejo integrado de pragas, que aumentam a proteção da cultura devido à interrupção dos ciclos de pragas, redução de surtos de pragas e principalmente evitam a erosão do solo (PRICE; LEVISTON, 2014; MORGAN et al., 2015; ADNAN et al., 2017a).

A segunda temática mais abordada dentre os artigos pesquisados foi o comportamento pró-ambiental relacionado à água (Tabela 2). A necessidade de se abordar esse tema reside no fato que a agricultura é um dos setores que mais demanda a utilização de água, todavia, por meio de práticas agrícolas sustentáveis é possível reduzir a quantidade de uso, o que torna essa problemática em proeminência para formuladores de políticas públicas (BJORNLUND et al., 2013).

Dois artigos enfocaram o comportamento pró-ambiental na agricultura conceitualizando a adoção de práticas agrícolas sustentáveis como estratégias de inovações tecnológicas (Tabela 2). Os estudos demonstraram que a difusão de ideias sustentáveis e inovadoras é facilitada por meio de demonstrações bem sucedidas, por aprendizagens coletivas e níveis mais altos de educação (GUEVARA-HERNANDEZ, 2017; BROWN; ROPER, 2017;). GuevaraHernandez (2017) ressalta que os agentes de extensão e outros (como os decisores políticos) devem possuir o conhecimento necessário para lidar e 
responder às exigências de diversidade com base na idade do produtor, gênero, nível socioeconômico e diferenças étnicas.

Apenas um estudo teve como temática a influência do tipo da propriedade (própria ou alugada) como preditora de atitudes pró-ambientais (Figura 2). De acordo com Arora et al. (2015), os proprietários de terras têm um foco de longo prazo para suas metas econômicas e sociais e prestam mais atenção aos objetivos pessoais. Enquanto os agricultores de terras alugadas se concentram em objetivos de curto prazo para garantir a maximização do lucro, bem como cumprir suas obrigações sociais. Essas diferenças de objetivos entre proprietários de terras e arrendatários influenciam o uso da terra, o uso de instrumentos financeiros e as atitudes ambientais.

\section{Metodologias em pesquisas sobre o comportamento pró-ambiental na agricultura}

O tamanho da amostra nos estudos variou de 20 a 9621 participantes prevalecendo as pesquisas com agricultores rurais, sendo que apenas duas fizeram comparação entre a população rural e urbana (SCHUPP; SHARP, 2012; BJORNLUND et al., 2013).

O referencial teórico mais utilizado foi a Teoria do Comportamento Planejado (TCP), de Ajzen e Fishbein (1980) em 23,5\% dos estudos. Essa teoria tem por base a compreensão de variáveis que tendem a predizer a possibilidade de um comportamento ocorrer. A intenção de realizar o comportamento é tratada como um antecedente direto do comportamento e é conduzida pelo controle comportamental percebido, pela atitude em relação ao comportamento e pelas normas subjetivas. As atitudes representam a avaliação das consequências percebidas do comportamento e da probabilidade de resultados, ao passo que as normas podem ser consideradas como padrões socialmente aceitos transmitidos por colegas, família e sociedade (AJZEN; FISHBEIN, 1980).

Outro modelo preditivo de práticas pró-ambientais baseado em estruturas psicológicas que teve destaque, foi a Teoria do Valor-CrençaNormas de Stern et al. (1998). Esse modelo postula que os comportamentos são uma forma de altruísmo guiado por normas morais e valores ambientais. Os valores são apresentados como características gerais relativamente estáveis, enquanto as crenças são consideradas mais específicas e focadas na natureza e nos resultados da relação humana com o ambiente.

Dentre os estudos selecionados, $41 \%$ empregaram instrumentos próprios, ou seja, elaborados pelos próprios autores para avaliar o comportamento pró-ambiental e 59\% utilizaram escalas padronizadas. Em relação aos instrumentos utilizados, a Escala de Valores Ambientais de Thompson e Barton (1994) foi a mais empregada e incluída em 17,64\% dos trabalhos. Os valores ambientais são definidos como um conjunto ordenado de

crenças sobre estados finais desejáveis que guiam a seleção ou avaliação de 
comportamentos ambientalmente relevantes e se distinguem entre três tipos de valores ambientais: ecocentrismo, antropocentrismo e apatia ambiental. Também teve destaque a Nova Escala de Medição de Autoeficácia (NGSENew General Self-Efficacy) de Chen, Gully e Eden (2001) que tem como objetivo definir a capacidade percebida de um indivíduo de se engajar em um comportamento (MORGAN et al., 2015).

Cabe ressaltar a metodologia usada por Guevara-Hernandez (2017) para compreender o desempenho, a promoção, a dinâmica e as limitações do Programa de Modernização Sustentável da Agricultura Tradicional (MasAgro), que empregou métodos quantitativos e qualitativos, como a entrevista estruturada e a implementação de oficinas participativas, baseando-se na Pesquisa-Ação, como forma de busca autorreflexiva de situações sociais e análise da realidade em estudo.

\section{Fatores pessoais e ambientais associados ao comportamento pró- ambiental na agricultura}

Cary e Roberts (2011) observaram, por meio de estudos de casos, que alguma forma de apoio governamental se fez necessária para garantir a adoção das práticas agrícolas sustentáveis. Muitas ferramentas de política financeira que encorajam a adoção voluntária de práticas de conservação baseiam-se na premissa de que os agricultores querem maximizar o interesse próprio, o que se mostra uma visão incompleta. Para Adnan et al. (2017b), embora a inovação das práticas agrícolas sustentáveis seja promovida e endossada por agências de extensão governamentais, pesquisas sugerem que a adoção entre agricultores é muito limitada. Essa limitação se deve ao fato que a agricultura envolve interface complexa entre influências privadas, sociais, financeiras e ambientais (MORGAN et al., 2015; SUMANE et al., 2018). Alinhar o gerenciamento ambiental com as disciplinas psicossociais é um passo necessário para alcançar resultados em prol do ambiente (FLORESS et al., 2017; JIANG et al., 2018).

Para Adnan et al. (2017b), a compreensão profunda dos fatores que determinam a intenção de utilizar as práticas agrícolas sustentáveis pode ajudar os formuladores de políticas a desenvolverem iniciativas para melhorar a viabilidade das taxas de adoção dessas práticas. Para cumprir este objetivo, é recomendado o estudo das características sociais e psicológicas dos agricultores, considerando o papel das motivações individuais e restrições contextuais do comportamento pró-ambiental (PRICE; LEVISTON, 2014; JIANG et al., 2018).

A tabela 3 representa as principais variáveis relacionadas às condições pessoais (intrínsecas) e contextuais (extrínsecas) que estão associadas ao comportamento pró-ambiental na agricultura. 
Tabela 3: Variáveis relacionadas às condições pessoais e contextuais/ambientais do comportamento pró-ambiental na agricultura

\begin{tabular}{ll}
\hline Variáveis & Porcentagem \\
\hline Conhecimento & $35,20 \%$ \\
Valores biosféricos & $29,40 \%$ \\
Autoeficácia & $11,70 \%$ \\
Orientação para o futuro & $5,80 \%$ \\
Consciência Ambiental & $5,80 \%$ \\
Valores extrínsecos & $5,80 \%$
\end{tabular}

Fonte: Autores (2020)

Como pode ser observado na tabela 3 , o conhecimento científico foi a variável identificada como mais promotora do comportamento estudado. Os resultados também mostraram que os valores biosféricos são um dos principais fatores que influenciam os comportamentos pró-ambientais na agricultura (BJORNLUND et al., 2013; PRICE; LEVISTON, 2014; HONING et al., 2015; HYNES; WILSON, 2016; BIJANI et al., 2017). Os valores biosféricos, expressos como preocupação por elementos da biosfera, como animais nativos, pássaros e plantas, têm relação direta com a prática de manejo próambiental da terra (NGUYEN et al., 2016; MARTIN; CZELLAR, 2017). Eles influenciam as crenças ambientais, tais como níveis percebidos de ameaça ambiental representados pela má qualidade da água, degradação da saúde do solo, erosão e ervas daninhas, que ativam normas pessoais comportamentais no cuidado da propriedade. A constatação de que os valores biosféricos podem aumentar os comportamentos pró-ambientais leva à consideração de como a formação de certos valores pode ser incentivada na tomada de decisão agrícola. Nesse sentido, Thogerson e Crompton (2009) destacam que, embora as estratégias voltadas para os resultados financeiros (variáveis extrínsecas) possam ser efetivas a curto prazo, elas tendem a produzir impactos negativos em longo prazo ao reforçar visões de mundo que são incompatíveis com ações ambientalmente sustentáveis. Os autores sugerem que haja o engajamento profundo que envolve a ativação de um conjunto de valores associados ao bem-estar coletivo e da comunidade, argumentando que mudanças no nível de valores resultarão em efeitos benéficos em vários domínios de comportamento pró-ambiental.

A orientação para o futuro, também se mostrou variável preditiva ao comportamento pró-ambiental na pesquisa de Morgan et al. (2015) com agricultores australianos. A Teoria da Perspectiva do Tempo (ZIMBARDO et al., 1997) sugere uma estrutura temporal através da qual os indivíduos geram coerência e significado a partir de eventos e experiências. Indivíduos orientados para 0 presente demonstram a necessidade de gratificação imediata, juntamente com a visão pessimista do futuro, enquanto os indivíduos 
orientados para o futuro têm maior probabilidade de retardar sua gratificação imediata na busca de objetivos futuros (HARBER et al., 2003).

O conhecimento científico foi a variável mais mencionada em relação à predição dos comportamentos pró-ambientais na agricultura (PRICE; LEVISTON, 2014; MORGAN et al., 2015; ADNAN et al., 2017a; BROWN; ROPER, 2017). De acordo com Brown e Roper (2017), a adoção de práticas de gestão ambiental e de novas tecnologias agrícolas foi maior em agricultores com formação universitária. Apesar da relevância dessa variável, nenhuma pesquisa foi realizada com estudantes, no entanto, a atenção a essa população torna-se indispensável, visto que estes serão produtores e/ou prestadores de assistência técnica, bem como responsáveis pelo incentivo à adoção de práticas sustentáveis.

O conceito de autoeficácia, ou seja, a capacidade percebida de um indivíduo de se engajar em um comportamento, apresentou-se como um importante preditor do comportamento pró-ambiental (MORGAN et al., 2015). Nesse sentido, a pesquisa realizada por Brown e Roper (2017) e Honig et al. (2015), revelou que os agricultores eram mais propensos a adotar práticas de gestão ambiental depois de ver uma demonstração bem-sucedida. Este fato chama a atenção para a necessidade de políticas públicas que promovam além do treinamento técnico-científico, o compartilhamento de experiências. Desta forma, os fatores sociais, dentre os quais devem ser consideradas as influências dos pares e dos grupos sociais para o comportamento próambiental, pois os processos de aprendizagem social são identificados como benéficos para o bem-estar dos agricultores e para os resultados ambientais (PRICE; LEVISTON, 2014; GARCÍA-LLORENTE et al., 2016).

Honig et al. (2015), com o objetivo investigar as condições favoráveis sob as quais a conservação da biodiversidade em propriedade privada é mais provável de ocorrer, avaliaram as motivações intrínsecas e extrínsecas, analisando a consciência ambiental, a motivação para participação no programa, os motivos que possibilitaram a implementação e as recompensas da adoção. Todos os participantes do estudo se referiram a algum nível de consciência ambiental e a maioria mencionou que esta foi um passo necessário para adoção das práticas de conservação. Dos participantes, $92 \%$ disseram ter sido motivados pela combinação de valores intrínsecos e extrínsecos para aderir ao programa de conservação, predominando os intrínsecos (86\%), como a responsabilidade de conservar e maior conexão com a natureza. Em relação às motivações extrínsecas foram citadas as voltadas para o valor, tais como: a redução de riscos, a melhoria da segurança no mercado por meio da diferenciação de produtos pela rotulagem e o desejo de obter vantagem competitiva (HONIG et al., 2015; MORGAN et al., 2015). Ademais, a respeito as condições favoráveis à conservação da biodiversidade por agricultores, os autores ressaltaram a necessidade de o governo prover o aprofundamento do conhecimento científico. Muitos referiram que antes do desenvolvimento do programa de conservação não adotavam práticas sustentáveis, pela falta de habilidades, ferramentas e/ou orientação. 
A agricultura envolve a interface complexa entre influências privadas, sociais, financeiras e ambientais (MORGAN et al., 2015; GARCíA-LLORENTE et al., 2016). É preciso considerar a integração entre as campanhas ambientais e a sociedade de maneira mais próxima, exigindo políticas horizontais. Os programas de intervenção agrícola precisam responder às questões ambientais e sociais e levar em conta os diversos valores e motivações dos proprietários de terras. Nesse sentido, as políticas públicas possuem um papel moderador em relação ao comportamento pró-ambiental ao estipularem leis específicas que devem estar voltadas à capacitação e formação dos sujeitos com programas de Educação Ambiental eficazes.

\section{Conclusões}

Os resultados mostraram que as principais variáveis que impeliram à promoção do comportamento pró-ambiental na agricultura foram de natureza intrínsecas, como os valores biosféricos, consciência ambiental, orientação para o futuro, autoeficácia e, com destaque, o conhecimento científico, que foi a variável mais identificada como promotora do comportamento estudado. Todavia, cabe ressaltar que a combinação dos valores intrínsecos e extrínsecos pode promover com mais eficiência as práticas sustentáveis na agricultura. Portanto, as políticas públicas devem favorecer e estimular esses comportamentos com leis de incentivos e intervenções formativas dos agricultores e/ou profissionais por meio de aprendizagem social de demonstrações bem-sucedidas de adoção de práticas agrícolas sustentáveis. Assim, se deve oportunizar cada vez mais a Educação Ambiental capaz de desenvolver a consciência ambiental fundamentada em valores biosféricos, visto que estes podem aumentar a adoção de comportamentos pró-ambientais na agricultura.

As variáveis relacionadas ao comportamento pró-ambiental na agricultura demonstraram a complexidade deste e constatam o desafio da interdisciplinaridade na agricultura, principalmente a da Psicologia Ambiental em conjunto com a Educação Ambiental para a construção de ferramentas eficazes e no desenho de políticas públicas mais pontuais para a promoção do comportamento pró-ambiental na agricultura.

\section{Agradecimentos}

Os autores agradecem ao Programa de Pós-Graduação em Tecnologias Limpas (PPGTL) da Universidade Cesumar, ao ICETI (Instituto Cesumar de Ciência, Tecnologia e Inovação) e à Université de Toulouse-Ecole d'Ingénieurs de Purpan et Pesquisador no Institut National de la RechercheAgronomique (INRA) - UMR Agroécologie, Innovations et Territoires, Toulouse - França pelo apoio ao desenvolvimento e promoção desta pesquisa. Os autores também agradecem à Coordenação de Aperfeiçoamento de Pessoal de Nível Superior Brasil (CAPES) e ao Instituto Cesumar de Ciência, Tecnologia e Inovação (ICETI) pelo apoio financeiro.

revista brasileira educação ambiental 


\section{Referências}

ADNAN, N. et al. Adoption of green fertilizer technology among paddt farmers: A possible solution for Malaysian food security. Land and Policy, v. 63, p. 3852, 2017a.

ADNAN, N.; NORDIN, S.M.; BIN ABU BAKAR, Z. Understanding and facilitating sustainable agricultural practice: A comprehensive analysis of adoption behavior among Malaysian paddy farmers. Land use policy, v. 68, p. 372-382, 2017b.

AJZEN, I.; FISHBEIN, M. Understanding Attitudes and Predicting Social Behaviour. Englewood Cliffs: Prentice-Hall, 1980.

ALCOCK, I. et al. Associations between pro-environmental behaviour and neighbourhood nature, nature visit frequency and nature appreciation: Evidence from a nationally representative survey in England. Environment International, v. 136, n. 1, p. 105441, 2020.

ARORA, P. et al. Ownership effect in the wild: Influence of land ownership on agribusiness goals and decisions in the Argentine Pampas. Journal of behavioral and experimental economics, v. 58, p. 162-170, 2015.

BERNSTEIN, J.; SZUSTER, B.W. The new environmental paradigm scale: Reassessing the operationalization of contemporary environmentalism. The Journal of Environmental Education, v. 50, n. 2, p. 73-83, 2019.

BEZERRA, Y.R.N.; FEITOSA, M.Z.S. A afetividade do agente comunitário de saúde no território: um estudo com os mapas afetivos. Ciência \& Saúde Coletiva, v. 23, p. 813-822, 2018.

BIJANI, M. et al. Pro-environmental analysis of farmers' concerns and behaviors towards soil conservation in central district of Sari County, Iran. International soil and water conservation research, v. 5, n. 1, p. 43-49, 2017.

BJORNLUND, H.; PARRACK, C.; DE LOE, R.C. Segmenting the Urban and Rural Populations of Southern Alberta for Improved Understanding of Policy Preferences for Water Reallocation. Society \& Natural Resources, v. 26, p. 1330-1350, 2013.

BROWN, P.; ROPER, S. Innovation and networks in New Zealand farming. Australian journal of agricultural and resource economics, v. 61, p. 422442, 2017.

CARY, J.; ROBERTS, A. The limitations of environmental management systems in Australian agriculture. Journal of environmental management, $v$. 92, n. 3, p. 878-875, 2011.

CHEN, G.; GULLY, S.M.; EDEN, D. Validation of a New General Self-Efficacy Scale. Organizational Research Methods, v. 4, n. 1, p. 62-83, 2001. 
CHIERRITO-ARRUDA, E. et al. Comportamento pró-ambiental e reciclagem: revisão de literatura e apontamentos para as políticas públicas. Ambiente \& Sociedade, v. 21, p. 1-18, 2018.

CLAYTON, S. et al. Expanding the role for psychology in addressing environmental challenges. American Psychologist, v. 71, n. 3, p. 199, 2016.

CORRAL, V. La definición del comportamiento proambiental. La Psicología Social en México, v. 8, n. 1, p. 466-472, 2000.

LIMA, G. C. O discurso da sustentabilidade e suas implicações para a educação. Ambiente \& Sociedade, Campinas, v. 6, n. 2, p. 99-119, dez. 2003.

FLORESS, K. et al. Toward a theory of farmer conservation attitudes: Dual interests and willingness to take action to protect water quality. Journal of Environmental Psychology, v. 53, p. 73-80, 2017.

FOOD AND AGRICULTURE ORGANIZATION OF THE UNITED NATIONS FAO. The state of food and agriculture 1995. Rome, Italy, 1995. ISBN 92-5103700-0.

Disponível

em: $<$ http://www.fao.org/docrep/017/v6800e/v6800e.pdf>. Acesso em: 30 set. 2018.

GARCÍA-LLORENTE, M. et al. Social farming in the promotion of socialecological sustainability in rural and periurban areas. Sustainability, v. 8, n. 12, p. 1238, 2016.

GOULD, R. K. et al. Environmental Behavior's Dirty Secret: The Prevalence of Waste Management in Discussions of Environmental Concern and Action. Environmental Management, v. 58, n. 2. p. 268-282, 2016.

GUEVARA-HERNANDEZ, F. Propuesta metodológica para el estudio de actores y estrategias de intervención tecnológica en Chiapas, México. Cultivos tropicales, v. 38, n. 2, p. 103-112, 2017.

HACKETT, P.MW. Modeling environmental concern: Theory and application. The Environmentalist, v.13, n. 2, p.117-120, 1993.

HARBER, K. D.; ZIMBARDO, P. G.; BODY, J. N. Participant self-selection biases as a function of individual differences in time perspective. Basic and Applied Social Psychology, v. 25, n. 3, p. 255-264, 2003.

HONING, M. et al. The conditions under which farmers are likely to adapt their behavior: A case study of private land conservation in the Cape Wine lands, South Africa. Land use policy, v. 48, p. 389-400, 2015.

HYLAND, J. J. et al. Farmers' perceptions of climate change: identifying types. Agriculture and Human values, v. 33, n. 2, p. 323-339, 2016.

INTERNATIONAL PANEL ON CLIMATE CHANGE - IPCC. Fifth assessment report. 2014. Disponível em: <http://www.ipcc.ch/report/ar5/index.shtml>. Acesso em: 30 jul. 2018. 
JIANG, L. et al. The impact of psychological factors on farmers' intentions to reuse agricultural biomass waste for carbon emission abatement. Journal of Cleaner Production, v. 189, n. 1, p. 797-804, 2018.

KLÖCKNER, C.A. A comprehensive model of the psychology of environmental behavior-A meta-analysis. Global Environmental Change, v.23. n. 5, p. 10281038, 2013.

MARTIN, C.; CZELLAR, S. Where do biospheric values come from? A connectedness to nature perspective. Journal of Environmental Psychology, v. 52, n. 1, p. 56-68, 2017.

MARTÍNEZ-SOTO, J. Comportamiento proambiental. Una aproximación al estudio del desenvolvimento sustentable con énfasis en el comportamiento persona-ambiente. Theomai, n. 99, 2004.

MOHER, D. et al. Preferred reporting items for systematic review and metaanalysis protocols (PRISMA-P) 2015 statement. Systematic Reviews, v. 4, n. 1, 2015.

MORGAN, M. I. et al. Landholder adoption of low emission agricultural practices: A profiling approach. Journal of Environmental Psychology, v. 41, p. 35-44, 2015.

NGUYEN, T. N.; LOBO, A.; GREENLAND, S. Pro-environmental purchase behaviour: The role of consumers' biospheric values. Journal of Retailing and Consumer Services, v. 33, p. 98-108, 2016.

ORGANISATION FOR ECONOMIC CO-OPERATION AND DEVELOPMENT, OECD. Environmental Performance Reviews: New Zealand. OECD Publishing, Paris, 2017.

PRICE, J.C.; LEVISTON, Z. Predicting pro-environmental agricultural practices: The social, psychological and contextual influences on land management. Journal of rural studies, v. 34, p. 65-78, 2014.

PROGRAMA DAS NAÇÕES UNIDAS PARA O DESENVOLVIMENTO - PNUD. Os Objetivos de Desenvolvimento Sustentável. Disponível em: $<$ http://www.pnud.org.br/ODS.aspx>. Acesso em: 10 jul. 2018.

RAMANKUTTY, N. et al. Trends in global agricultural land use: implications for environmental health and food security. Annual review of plant biology, v. 69, n. 1, p. 789-815, 2018.

SCHUPP, J.L.; SHARP, J.S. Exploring the social bases of home gardening. Agriculture and human values, v. 29, p. 93-105, 2012.

SONCINI, A.; PIERINI, A.J.; GALLO, Z. A compreensão dos alunos ao primeiro ano de Agronomia sobre Agroecologia: uma análise do presente e perspecticas de futuro. Retratos de Assentamentos, v. 19, n. 2, 2016.

STERN, P.C.; DIETZ, T.; GUAGNANO, G.A. A brief inventory of values. Educational and psychological measurement, v.58, p. 984-1001, 1998. 
SUMANE, S. et al. Local and farmers' knowledge matters! How integrating informal and formal knowledge enhances sustainable and resilient agriculture. Journal of Rural Studies, v. 59, p. 232-241, 2018.

SWAMI, V. et al. Egoistic, altruistic, and biospheric environmental concerns: a path analytic investigation of their determinants. Scandinavian Journal of Psychology, v. 51, n. 2, p. 139-45, 2010.

SZUMELDA, A.U. Agriculture and everyday realities on small farms-An entrepreneurial challenge to farmers between the desire for autonomy and a secure existence. Two examples from east and south-east Poland. Journal of Rural Studies, v. 67, n. 1, p. 57-68, 2019.

THOGERSON, J.; CROMPTON, T. Simple and painless? The limitations of spillover in environmental campaigning. Journal of Consumer Policy, v. 32, p. 141-146, 2009.

THOMPSON, S.C.G.; BARTON, M.A. Ecocentric and anthropocentric attitudes toward the environment. Journal of Environmental Psychology, v. 14, n. 2, p. 149-157, 1994.

WU, D. W; DIGIACOMO, A.; KINGSTONE, A. A Sustainable Building Promotes Pro-Environmental Behavior: An Observational Study on Food Disposal. PLoS ONE, v. 8, n. 1, 2013.

ZACARIAS, E.F.J.; HIGUCHI, M.I.G. Relação pessoa-ambiente: caminhos para uma vida sustentável. Interações (Campo Grande), Campo Grande, v. 18, n. 3, p. 121-129, Sept. 2017.

ZEWELD, W. et al. Smallholder farmer's behavioural intentions towards sustainable agricultural practices. Journal of Environmental Management, $v$. 187, p. 71-81, 2017.

ZIMBARDO, P.G.; KEOUGH, K.A.; BODY, J.N. Present time perspective as a predictor of risky driving. Personality and Individual Differences, v. 23, n. 6, p. 1007-1023, 1997. 